\title{
Study of Mean platelet volume in type 2 diabetes mellitus
}

\author{
El HussienyYousef Al Refaee M.B.B.CH/ Prof. Hassan Ahmad Hassanien MD \\ Prof. Ahmad MohamadBoghdady MD/Dr. HamdySaadMohamad MD
}

Internal medicine department, faculty of medicine, Sohag University

\begin{abstract}
Background: Altered platelet morphology and function have been reported in patients with diabetes mellitus. They are likely to be associated with the pathological processes and increased risk of vascular disease seen in these patients. We aimed to determine the mean platelet volume (MPV) in diabetics compared to nonediabetics, to see if there is a difference in MPV between diabetics with and without macro and microvascular complications, and to determine the correlation between MPVand fasting blood glucose, glycosylated hemoglobin (HbA1c), patient age, and duration of diabetes, respectively.

Methods: We measured MPV in 100 consecutive Type 2 diabetic patients and 50 non diabetic control subjects without known coronary artery disease who had complete blood count, FBG, HbA1c, serum creatinine and ALB/Creat ratio. Statistical evaluation was performed by SPSS for Windows statistics programme using multivariate logistical regression analysis.

Results: MPV was significantly higher in diabetics compared to age- and sex-matched nonediabetic healthy controls, significantly higher with complicated DM compared to none complicated DM, and significantly higher with poor glycemic control compared to good glycemic control.

Conclusions: Our results show significantly higher MPV in diabetic patients than in the nonediabetic controls. This suggests that platelets may play a role in the micro and macrovascular complications of diabetic patients.
\end{abstract}

\section{Introduction}

Diabetes mellitus is a complex disease characterized by chronic hyperglycemia responsible for complications affecting the kidneys, eyes, peripheral nerves, and micro and macro vascular systems $\boldsymbol{Z}$ Hekimsoya, et al., (2004). Platelet hyperactivity is accompanied by an increased synthesis of thromboxane and/or a decreased prostacyclin productionRollini, et al., (2013).Larger platelets are more reactive and able to aggregate. They contain denser granules, secrete more serotonin and bthromboglobulin, and produce more thromboxane A2 than smaller platelets E.Y. Lee, et al., (2013). This indicates that changes in the platelet count and
MPV reflect the state of thrombogenesis.This suggests a relationship between platelet function and diabetic complications (Srivastava, etal., 1994; Kim, et al., 1995; Bath \& Butterworth, 1996; Mazzanfi\&Mutus, 1997).Platelet volume is a marker of platelet function and activation. It is measured as mean platelet volume (MPV) by clinical hematology analyzers Bath \& Butterworth, (1996).

In this study we aimed to(1) compare the MPV in diabetics with that in age- and sex-matched non diabetic healthy controls, (2) see whether there is a difference in MPV between diabetic patients with and without macro and 
micro vascular complications, (3) determine the correlation between MPV and glycemic control.

\section{Patients and methods}

The study group included 100 consecutive Type 2 diabetic patients (49 females and 51 males); mean age was 49 \pm 14 years (range 23-77 years). The mean duration of diabetes was $8 \pm 4.8$ years (range 1-21 years). Diabetic patients were evaluated clinically and laboratory to detect macro and micro vascular complications, other cardiovascular risk factors, type of treatment and glycemic state by measuring $\mathrm{FBG}, \mathrm{HbA} 1 \mathrm{c}, \mathrm{CBC}$, serum

\section{Results}

1-Table shows significant increase in chronic diabetic vascular complications among diabetic patients versus non diabetics $(p<0.0001)$ and also significant increase in complications with poor glycemic control versus good glycemic control $(\mathrm{p}=0.01)$.

\begin{tabular}{|l|c|c|c|}
\hline Complications & Controlled DM & Uncontrolled DM & Controls \\
\hline No & $15(30.00 \%)$ & $5(10.00 \%)$ & $50(100 \%)$ \\
Yes & $35(70.00 \%)$ & $45(90.00 \%)$ & 0 \\
\hline \multicolumn{3}{|c|}{$P<0.0001, P 1=0.01, P 2<0.001, P 3<0.001$} \\
\hline
\end{tabular}

2-Tableshows significant increase in MPV among diabetic patients versus non diabetics $(\mathrm{p}<0.0001)$ and also significant increase in MPV with poor glycemic control versus good glycemic control $(\mathrm{p}<0.0001)$.

\begin{tabular}{|l|c|c|c|}
\hline MPV(fl) & Controlled DM & Uncontrolled DM & Controls \\
\hline Mean \pm SD & $12.62 \pm 1.38$ & $14.08 \pm 1.16$ & $9.04 \pm 1.35$ \\
Median (range) & $12.5(10-15)$ & $14(12-16)$ & $9(7-11)$ \\
\hline \multicolumn{3}{|c|}{$\mathbf{P}<\mathbf{0 . 0 0 0 1}, \mathbf{P} 1<\mathbf{0 . 0 0 0 1}, \mathbf{P} 2<\mathbf{0 . 0 0 0 1}, \mathbf{P 3}<\mathbf{0 . 0 0 0 1}$} \\
\hline
\end{tabular}


3-Tableshows significant increase in incidence of chronic vascular complications related to DM with increase in MPV ( $\mathrm{p}=0.0002)$.

\begin{tabular}{|l|c|c|c|}
\hline MPV(fl) & Complication & No complications & P value \\
\hline Mean \pm SD & $13.58 \pm 1.36$ & $12.13 \pm 1.41$ & 0.0002 \\
Median (range) & $14(11-16)$ & $12(10-15)$ & \\
\hline
\end{tabular}

4-Tableshows significant relationship between MPV and type of treatment of DM $(\mathrm{p}=0.0071)$ as mean MPV with insulin therapy significantly lower than OHG therapy, also shows significant relationship between MPV and HTN $(p=0.002)$ as mean significantly increased MPV with presence of HTN.Also shows MPV with males higher than females but not significant $(\mathrm{p}=0.57)$.Also shows MPV with smokers higher than non-smokers but not significant $(\mathrm{p}=0.53)$.

\begin{tabular}{|l|c|c|}
\hline Variable & Mean MPV (fl) \pm SD & P value \\
\hline Gender Females & $13.27 \pm 1.48$ & 0.57 \\
Males & $13.43 \pm 1.46$ & \\
\hline Treatment Insulin & $12.29 \pm 1.50$ & 0.0071 \\
OHG & $13.40 \pm 1.45$ & \\
\hline HypertensionNo & $12.94 \pm 1.39$ & 0.002 \\
Yes & $13.83 \pm 1.42$ & \\
\hline Smoking No & $13.29 \pm 1.43$ & 0.53 \\
Yes & $13.5 \pm 1.58$ & \\
\hline
\end{tabular}


5-Tableshows significant increase in MPV with aging $(\mathrm{p}=0.04)$, no significant increase inMPV with prolongation of duration of DM $(p=0.13)$, significant increase in MPV with increased FBG \& HbA1c as mean poor glycemic control $(\mathrm{p}<0.0001)$.

\begin{tabular}{|l|c|c|}
\hline Variable & Correlation co-efficient (r) & P value \\
\hline Age/years & 0.20 & 0.04 \\
\hline Duration of DM & 0.15 & 0.13 \\
\hline FBG & 0.55 & $<0.0001$ \\
\hline HBA1c & 0.63 & $<0.0001$ \\
\hline
\end{tabular}

NB. $\mathrm{P}$ compared the 3 groups, p1 compared controlled DM with uncontrolled DM, p2 compared controlled DM with controls, p3 compared uncontrolled DM with controls.

\section{Discussion}

Platelet hyperactivity in DM may be one factor in the severe and profound vasculopathies that are so often associated with this disorder. There are studies that have shown increased platelet aggregation in diabetes mellitus, and this may have a role in its vascular complications (Heath, et al, 1971; Halushka, et al, 1977). Activated platelets respond to activated leukocytes and endothelial cells via adhesion molecules linking inflammation and thrombosis.

The first evidence of a significant, positive association between MPV and diabetes was published by P.C Sharpe and Trinick (1993), who described that MPV was increased in persons with diabetes compared with non-diabetic subjects. In a following investigation, $\boldsymbol{Z}$. Hekimsoy, et al. (2004) measured MPV in 145 consecutive patients with type 2 diabetes and 100 non-diabetic controls, and found that MPV was higher and the mean platelet count was lower among those with diabetes than among nondiabetic healthy controls. Similarly, two small case-control studies have confirmed that MPV was significantly higher in patients with diabetes than in those without this condition $(N$. Papanas, et al. 2004; E. Coban, et al. 2006). More recently, T.A.Kodiatte, et al. (2012) assessed MPV and platelet count in 300 type 2 diabetic patients and 300 non-diabetic subjects, and found that there were significant positive associations of MPV with levels of fasting glucose, postprandial glucose and $\mathrm{HbA1c}$. Finally, in a small case-control study involving 366 diabetic patients and 54 healthy controls, E.Y.Lee, et al. (2013) confirmed that both MPVand immature platelet fraction were significantly elevated in persons with diabetes compared with control subjects. To date, relationship of MPV with DM never previously studied in Sohag University hospital and our community. Our study showed the following:

1. Significant increase in MPV with diabetes mellitus.

2. Significant increase in MPV with poor glycemic control.

3. Significant increase in vascular complications of DM with higher MPV.

4. Variable degrees of relationship among MPV and different cardiovascular risk factors as age, gender, smoking, hypertension. 
5. Significant relationship between MPV and type of treatment of DM (insulin versus OHG), MPV higher with OHG therapy versus insulin therapy.

In our study, diabetic patients had significantly larger MPV than nondiabetic controls. Almost all previous studies agreed with this result (P.C. Sharpe and Trin ık 1993; Z. Hekimsoy, et al. 2004; Giuseppe Lippi, et al. 2015; NakarinSansanayudh, et al. 2016).

The mechanism of increased platelet size in DM is osmotic swelling due to raised blood glucose and due to raised levels of some glucose metabolites Martyn, et al., (1986). There was another possible explanation for the increased platelet size. Some studies have shown a shorter life span for platelets in diabetics (Jones, et al., 1981; Tindall, et al, 1981). But now there is a consensus that platelet size is not related to platelet age and platelet size is determined at the time of production from the megakaryocyte Martin, (1989).

In our study, we found a significant relationship between glycemic control and vascular complications of DM and MPV as mean poor glycemic control (high FBG and HbA1C) lead to higher MPV lead to more macro and micro vascular complications.

Older studies disagreed with this result as (P.C. Sharpe and Trinick 1993, Z. Hekimsoy, et al. 2004), these studies see that there is no correlation between MPV and HbA1c, fasting blood glucose, patient age, and duration of diabetes. This suggests that the increase in MPV may be due to the diabetic state alone. But recent studies approved that there is a strong relationship between the previous three variables (Giuseppe Lippi, et al. 2015; NakarinSansanayudh, et al. 2016).

We found almost no association between
MPV and age of patient which was in agreement with some previous studies (Demirin, et al. 2011, Maluf, et al. 2014). However, one large study reported the association between age and MPV only in men, but not in women Santimone, et al, (2011). The large study of MPV and age, adjusting for other variables and including a wide range of ages, should be encouraged to confirm the association between MPV and age.

Gender influence on MPV is another topic for debate. Shimodaira, et al. (2014), reported larger MPV in male, whereas most previous studies found no difference in MPV between male and female Butkiewicz, et al. (2006). Conversely in our study, MPV was higher in males than females but not significant. One possible explanation might be the racial difference in populationstudied. Maluf, et al. (2014), reported substantial difference in MPV among races .The different studies of association between MPV and risk factors such as sex and age were conducted in different ethnic groups. The future study of reference interval and influence of other risk factors on MPV in different ethnicity is required.

The association between MPV and smoking was detected in univariate analysis but disappeared after adjusting with other variables in some previous studies. Despite a report of an increase in MPV over time in passive smoker who were exposed to moderate to high intensity of cigarette smoke in one small experimental study Yarlioglues, et al. (2012), most evidence form observation studies Biljak, et al, (2011) showed no association between smoking status and MPV which is consistent with the results from our study.

Our study suggested similar effects of 
hypertension on MPV as diabetes, i.e., subjects with hypertension had approximately 0.9fL larger MPV than normotensive subjects. This corresponded with previous publications Ntaios, et al. (2011).

Our study suggested no significant relationship between MPV and duration of DM as mean that the increase in MPV occurs at the beginning of the disease and persists for its duration. So the increase in incidence of diabetic vascular complications with longer duration due to other risk factors as HTN, smoking, obesity, dyslipidemia, poor glycemic control but this not exclude thevery important role of MPV as indicator and risk stratification factor of macro and micro vascular complications of DM, (Z.Hekimsoy, et al, 2004; Tavil, et al, 2007).

In our study, MPV was higher in diabetics with oral hypoglycemic treatment than in diabetics with insulin therapy. Insulin by achieving good glycemic control and thereby keeping MPV low is playing some role in preventing vascular complications Vernekar, et al. (2013).

Vernekar, et al, (2013) and our results showed that early initiation of insulin treatment in confirmed cases of Type 2 diabetics not only may help in controlling blood glucose level but also may help in keeping MPV low and thereby preventing possibility of impending acute vascular events.

\section{Conclusion}

In type 2 diabetes mellitus MPV is increased and it is indicative of worsening glycemic control. Increased platelet size may be one factor in the increased risk of atherosclerosis associated with diabetes mellitus and associated vascular complications. Hence, MPV would be a useful prognostic marker of macro-micro vascular complications in diabetes There are relationships among MPV and other cardiovascular risk factors as age, gender, HTN and smoking as described above. Early initiation of insulin treatment in confirmed cases of Type 2 diabetics not only helps in controlling blood glucose level but also helps in keeping MPV low and thereby preventing possibility of impending vascular events.

\section{References}

1. Bath P M \& Butterworth R J, 1996. Platelet size: measurement, physiology and vascular disease. Blood Coagulation and Fibrinolysis, 7 (2), 157- 161.

2. Biljak VR, et al. Platelet count, mean platelet volume and smoking status in stable chronic obstructive pulmonary disease. Platelets 2011; 22(6):466-70.

3. Butkiewicz AM, et al. Platelet count, mean platelet volume and thrombocytopoietic indices in healthy women and men. Thromb Res 2006; 118(2):199-204.

4. Demirin $\mathrm{H}$, et al. Normal range of mean platelet volume in healthy subjects: insight from a large epidemiologic study. Thromb Res 2011; 128(4):358-60.

5. E. Coban, F. Bostan, M. Ozdogan, The mean platelet volume in subjects with impaired fasting glucose, Platelets 17 (2006) 67-69.

6. E.Y. Lee, S.J. Kim, Y.J. Song, et al, Immature platelet fraction in diabetes mellitus and metabolic syndrome, Thromb . Res. 132 (2013) 692-695.

7. Giuseppe Lippi ,Gian Luca Salvagno ,Antonio Nouvenne, et al, The mean platelet volume is significantly associated with higher glycated 
hemoglobin in a large population of unselected outpatients, primary care diabetes 9 (2015 ) 226-230.

8. Halushka P. V., Lurie D., \& Colwell J. A. (1977). Increased synthesis of prostaglandin-E-like material by platelets from patients with diabetesmellitus. New England Journal of Medicine, 297, 1306- 1310.

9. Heath H., Bridgen W D., Canever J V, 1971. Platelet adhesiveness and aggregation in relation to diabetic retinopathy. Diabetologia, 7, 308-315.

10. Jones R. L., Paradise C \& Peterson CM, 1981. Platelet survival in patients with diabetes mellitus. Diabetes, 30, 486489.

11. Kim SW., Ryu GH., Lee I., et al (1995). Adhered platelet morphology in diabetes mellitus. Diabetes and Metabolism, 21 (1), 50- 53.

12. Maluf CB, Barreto SM, Vidigal PG. Standardization and reference intervals of platelet volume indices: Insight from the Brazilian longitudinal study of adult health (ELSABRASIL). Platelets 2014:1-8.

13. Martin J. F. (1989). The relationship between megakaryocyte ploidy and platelet volume. Blood Cells, 15, 108117.

14. Martyn C N., Matthews D M., PoppSnijders, et al, 1986. Effects of sorbinil treatment on erythrocytes and platelets of persons with diabetes. Diabetes Care, 9, 36- 39 .

15. Mazzanfi, L, \&Mutus, B. (1997). Diabetes-induced alterations in platelet metabolism. Clinical Biochemistry, 30 (7), 509-515

16. N. Papanas, G. Symeonidis, E. Maltezos, et al., Mean platelet volume in patients with type 2 diabetes mellitus, Platelets 15 (2004) 475-478.

17. NakarinSansanayudh,

DittapolMuntham, SukitYamwong, et al: The association between mean platelet volume and cardiovascular risk factors, European Journal of Internal Medicine 30 (2016) 37-42.

18. Ntaios G, et al. Hypertension is an independent predictor of mean platelet volume in patients with acute ischemic stroke. Intern Med J 2011; 41 (9):691-5.

19. P.C. Sharpe, T. Trinick, Mean platelet volume in diabetes mellitus, Q. J. Med. 86 (1993).

20. Rollini F, et al. Platelet function profiles in patients with diabetes mellitus. J Cardiovascular Transl Res 2013;6(3):329

21. Santimone I, et al. White blood cell count, sex and age are major determinants of heterogeneity of platelet indices in an adult general population: results from the MOLI-SANI project. Haematologica 2011; 96(8):1180-8.

22. Shimodaira M, et al. Gender differences in the relationship between serum uric acid and mean platelet volume in a Japanese general population. Platelets 2014; 25(3): 202-6.

23. Srivastava S., Joshi C. S., Sethi P. P, et al 1994. Altered platelet functions in non-insulin-dependent diabetes mellitus (NIDDM). Thrombosis Research, 76 (5), $451-461$.

24. T.A. Kodiatte, U.K. Manikyam, S.B. Rao, et al., Mean platelet volume in type 2 diabetes mellitus, J. Lab. Phys. 4 (2012) 5-9.

25. Tavil Y, Sen N, Yazici HU, et al. Mean platelet volume in patients with metabolic syndrome and its relationship 
with coronary artery disease. Thromb Res 2007; 120:245-250.

26. Tindall H., Paton R. C., Zuzel M., et al 1981. Platelet life span in diabetics with and without retinopathy. Thrombosis Research, 21, 641- 648.

27. Vernekar PV, Vaidya KA .Comparison of mean platelet volume in type 2 diabetics on insulin therapy and on oral hypoglycemic agents. J Clin Diabetes Res 2013; 12:2839-2840.

28. Yarlioglues $M$, et al. The acute effects of passive smoking on mean platelet volume in healthy volunteers. Angiology 2012; 63(5):353-7.

29. Z. Hekimsoy, B. Payzin, T. Ornek, et al, Mean platelet volume in type2 diabetic patients, J. Diabetes Complications 18 (2004)173-176. 